\title{
1980: Du sens pratique de gravir une échelle. Bourdieu et Ducrot sur le toit du monde
}

Jérôme Jacquin

\section{(2) OpenEdition}

Journals

Édition électronique

URL : http://journals.openedition.org/edl/2367

DOI : $10.4000 /$ edl. 2367

ISSN : 2296-5084

Éditeur

Université de Lausanne

\section{Édition imprimée}

Date de publication : 15 mars 2020

Pagination : 99-102

ISBN : 978-2-940331-73-4

ISSN : 0014-2026

\section{Référence électronique}

Jérôme Jacquin, «1980: Du sens pratique de gravir une échelle. Bourdieu et Ducrot sur le toit du monde », Études de lettres [En ligne], 312 | 2020, mis en ligne le 24 mars 2020, consulté le 17

septembre 2020. URL : http://journals.openedition.org/edl/2367 ; DOI : https://doi.org/10.4000/edl. 2367

\section{(c) Études de lettres}




\section{0: DU SENS PRATIQUE DE GRAVIR UNE ÉCHELLE. BOURDIEU ET DUCROT SUR LE TOIT DU MONDE}

En 1980, deux sommités des sciences humaines et sociales, deux chercheurs affiliés à l'École des hautes études en sciences sociales (EHESS), deux personnes nées en 1930 à quelques semaines d'intervalle, publient, chacun de son côté, mais tous deux aux éditions de Minuit, un ouvrage décisif.

Dans Le sens pratique ${ }^{1}$, le sociologue Pierre Bourdieu scrute et discute la manière dont les agents sociaux se fondent sur un sens pratique pour gérer, de manière plus ou moins ritualisée, plus ou moins consciente, plus ou moins logique, leur vie quotidienne et plus généralement leur rapport au monde. Ces agents, vous et moi faisons au mieux, développons un certain savoir-faire au sujet du social pour en faire un «allant de soi $»^{2}$ familier. En d'autres termes, le sens pratique rend tangible notre marge de manœuvre, entre les forces plus contraignantes de l'habitus et l'imprévisibilité des situations dans lesquelles nous sommes continuellement plongés. Ainsi trouve-t-on chez Bourdieu la métaphore du «jeu», des "coups» qui se succèdent et qui dessinent, rétrospectivement, l'apparence d'une trajectoire ${ }^{3}$.

I. L'auteur de cette courte notice n'a ni l'objectif ni la compétence de véritablement résumer ce riche ouvrage à facettes; il se contente d'en tirer les quelques éléments qui lui paraissent les plus éclairants du moment où l'on cherche à les mettre en rapport avec le travail d'O. Ducrot.

2. Selon l'expression d'A. Schütz (Collected Papers, I, p. 77).

3. "Le sens pratique oriente des "choix" qui pour n'être pas délibérés n’en sont pas moins systématiques, et qui, sans être ordonnés et organisés par rapport à une fin, 
À première vue bien éloigné de ce genre de considérations, Oswald Ducrot publie au même moment Les échelles argumentatives. Le linguiste y décrit la richesse et la complexité du langage et identifie en particulier des séries d'unités linguistiques qui se laissent moins résumer en termes descriptifs (les concepts de "pomme», de "table», de "gris») qu'instructionnels. Ainsi, si on compare "peu» ("Il me reste peu d'argent») et "un peu» ("Il me reste un peu d'argent»), on constate que ces deux expressions peuvent référer à une même quantité, objective, de liquidités, mais qu'elles diffèrent au niveau du type d'instruction, autrement dit de conclusion, à inférer. Dans le premier cas, le locuteur exprime une insatisfaction à laquelle remédier, tandis que dans le second le locuteur s'en accommode. L'« orientation argumentative» diffère, et choisir l'une ou l'autre expression manifeste la marge de manœuvre qui s'offre au sujet parlant qui, bien loin de subir unilatéralement une langue élaborée par d'autres, se voit offrir dans cette même langue des occasions d'agir:

Pour se justifier de ne pas s'occuper d'une affaire, on pourra dire qu'on est peu inquiet sur le résultat, mais non pas qu'on l'est un peu ${ }^{4}$.

On peut à certains égards regretter que la rencontre entre ces deux pensées n'ait pas eu lieu. Cela doit probablement au fait que Bourdieu publiera deux ans plus tard Ce que parler veut dire: l'économie des échanges linguistiques, qui remet en cause la notion de performativité, le sociologue reprochant plus généralement aux linguistes de travailler sur un objet décontextualisé (la langue), abstrait des conditions sociales d'emploi, et ce faisant de contribuer à une fétichisation de la langue et à son élévation et rigidification au rang de langue d'État. Le dialogue paraît donc plus que compromis, dans la mesure où langue et société sont disjointes, renvoyées dos à dos, avec l'idée d'une prévalence nette de la seconde sur la première. Pour le Bourdieu de 1982, les mots importent peu, voire aucunement, du moment où les conditions sociales ne sont pas réunies.

Pourtant, à la lumière des publications de 1980 et au-delà des différences de sensibilité entre les deux auteurs concernant les rapports complexes entre langue et société, une rencontre était non seulement

n'en sont pas moins porteurs d'une sorte de finalité rétrospective» (P. Bourdieu, Le sens pratique, p. 111).

4. O. Ducrot, Les échelles argumentatives, p. 24. 
envisageable, mais aussi prometteuse. Ces deux œuvres, qui peuvent à bien des égards être élevées au rang d'emblèmes d'un structuralisme d'avant la chute et la disgrâce, montrent bien que l'analyse des structures, des plus sociales aux plus linguistiques, n'est pas incompatible avec l'idée de marge, de dynamisme, de potentialités et donc d'agentivité, ce qui leur a pourtant été largement reproché par la suite, à l'une comme à l'autre. Avec cette approche des systèmes en tant que ressources incorporant des potentialités d'action, le structuralisme restait prometteur pour poursuivre l'étude pragmatique du rapport des formes à leur contexte d'émergence: les structures, loin de constituer de rigides canevas étouffant l'individu, présentent des alternatives qui sont autant de coups à jouer, d'occasions à saisir, de décisions à prendre, de trajectoires à dessiner, de positions à endosser au sein d'un tout qui, qu'on le veuille ou non, contribue à donner du sens. On peut dès lors regretter que la vague constructiviste qui a suivi, et qui se trouve aujourd'hui dans une sorte d'impasse à trop vouloir rendre compte de toutes les spécificités contextuelles en perdant parfois de vue le panorama général des formes disponibles et des forces en jeu, ait rendu scientifiquement désuète une pensée si riche, féconde et pourvoyeuse de résultats systématiques.

Jérôme JACQUIN

Section des sciences du langage et de l'information, Faculté des lettres, Université de Lausanne

\section{BIBLIOGRAPHIE}

Bourdieu, Pierre, Le sens pratique, Paris, Minuit, 1980.

—, Ce que parler veut dire: l'économie des échanges linguistiques, Paris, Fayard, 1982.

Ducrot, Oswald, Les échelles argumentatives, Paris, Minuit, 1980.

Scнütz, Alfred, Collected Papers I: The Problem of Social Reality, The Hague, Martinus Nijhoff, 1962. 
